\title{
Methylation of DIRAS1 promotes colorectal cancer progression and may serve as a marker for poor prognosis
}

\author{
Ruipan Zheng ${ }^{1,2,3}$, Dan Gao ${ }^{1,3}$, Tao He ${ }^{1}$, Meiying Zhang ${ }^{1,3}$, Xiaomei Zhang ${ }^{1}$, Enqiang Linghu', Lixin Wei ${ }^{*}$
} and Mingzhou Guo ${ }^{1 *}$ (D)

\begin{abstract}
Background: DIRAS1 is a new member of the Ras gene family. It was described as a potential tumor suppressor in human glioblastomas and esophageal cancer. The role of DIRAS1 in colorectal cancer remains unclear.

Methods: To explore the epigenetic changes and function of DIRAS1 in human colorectal cancer, we studied ten colorectal cancer cell lines and 146 primary colorectal cancer samples and 50 matched adjacent samples using semi-quantitative reverse transcription PCR, immunohistochemistry, methylation-specific PCR and bisulfite sequencing, western blot, flow cytometry, and transwell assays.

Results: DIRAS1 expression was found in DKO and HCT116 cells, while reduced expression was detected in LoVo, SW48, LS180, and SW620 cells, and there was no expression detected in DLD1, HT29, RKO, and SW480 cells. Complete methylation was found in the promoter region of DLD1, HT29, RKO, and SW480 cells. Partial methylation was detected in LoVo, LS180, SW48, and SW620 cells, and unmethylation was found in DKO and HCT116 cells. These results indicate that promoter region methylation correlated with loss of/reduced expression of DIRAS1. Re-expression of DIRAS1 was induced by 5-aza-2'-deoxycytidine, suggesting that the expression of DIRAS1 is regulated by promoter region methylation. DIRAS1 was methylated in $47.3 \%$ (69/146) of primary colorectal cancer samples, no methylation was found in non-cancerous colonic tissue samples. Methylation of DIRAS1 was significantly associated with TNM stage $(P<0.05)$ and short survival time $(P=0.0121)$. DIRAS1 induced apoptosis and inhibited cell proliferation, migration, and invasion in colorectal cancer. Finally, DIRAS1 suppressed colorectal cancer cell xenograft growth in nude mice.
\end{abstract}

Conclusions: DIRAS1 is frequently methylated in human colorectal cancer and the expression of DIRAS1 is regulated by promoter region methylation. Methylation of DIRAS1 is a marker of poor prognosis in human colorectal cancer.

Keywords: DIRAS1, Epigenetics, DNA methylation, Colorectal cancer

\section{Background}

Colorectal cancer (CRC) is the third most common malignancy and the fourth leading cause of cancer-related death worldwide [1-3]. Genetic alterations are common in CRC cells, while they are rare in normal cells [4]. Hereditary forms of colorectal cancer account for less than $5 \%$ of colorectal cancer [5]. Inactivation of gene function by germline mutations within at least one of four

\footnotetext{
*Correspondence: weilx301@263.net; mzguo2014@163.com

${ }^{2}$ Department of Pathology, Chinese People's Liberation Army General

Hospital, 28 Fu-Xing Road, Beijing 100853, China.

'Department of Gastroenterology and Hepatology, Chinese People's

Liberation Army General Hospital, \#28 Fuxing Road, Beijing 100853, China

Full list of author information is available at the end of the article
}

mismatch repair genes (MLH1, MSH2, MSH6, and PMS2) can be found in approximately $70 \%$ of cases, and $95 \%$ of the mutations occur in hMSH2 or hMLH1 [6]. In sporadic cancer, DNA damage repair genes are rarely found to be mutated. Researchers have confirmed that promoter region methylation accounts for $80-90 \%$ of MLH1 inactivation in sporadic MSI-H (high-level microsatellite instability) colorectal cancer [7]. Thus, DNA methylation is increasingly recognized as a common mode of gene inactivation in DNA damage repair and other signaling pathways. Different genes were found to be frequently methylated in human colorectal cancer [8-11]. Some studies demonstrated that somatic 
epigenetic dysregulation occurs not only in cancer tissues but also in noncancerous and preneoplastic tissues. These studies suggest that epigenetic events are potentially more promising somatic CRC risk markers than gene mutations. Age and environmental factors are regarded as impact factors of epigenetics [4].

DIRAS1 is a distinct subfamily member of Ras GTPases. DIRAS1 is also known as Rig (Ras-related inhibitor of cell growth), which is located in chromosome 19p13.3 [12]. DIRAS1 has been described as a potential tumor suppressor in human glioblastomas and esophageal cancer, and downregulation of the DIRAS1 predicts poor prognosis in esophageal squamous cell carcinoma [12, 13]; however, its role in colorectal cancer remains unclear. Therefore, we analyzed the epigenetic regulation and function of DIRAS1 in human colorectal cancer.

\section{Methods}

\section{Cell lines and tumor specimens}

Human colorectal cancer lines, DKO (DNMT1 and DNMT3b double knockout from HCT116 cells, a generous gift from Stephen Baylin), DLD1, HCT116, HT29, LoVo, LS180, RKO, SW48, SW480, and SW620 cells were previously established from primary colorectal cancer tissue samples and cultured as described previously [10].

Primary colorectal cancer samples (146) and matched adjacent samples (50) were collected during surgical resection at the Chinese PLA General Hospital. Twenty-six cases of formalin-fixed paraffin-embedded tumor tissues were available with matched adjacent tissue samples. All samples were collected under the guidelines approved by the institutional review board of the Chinese PLA General Hospital.

\section{5-Aza-2'-deoxycytidine treatment}

Colorectal cancer cell lines were split to a low density (30\% confluence) $12 \mathrm{~h}$ before treatment. Cells were treated with 5-aza-2'-deoxycytidine (DAC, Sigma, St. Louis, MO) at a concentration of $2 \mu \mathrm{M}$. Growth medium conditioned with DAC at a concentration of $2 \mu \mathrm{M}$ was exchanged every $24 \mathrm{~h}$ for a total of $96 \mathrm{~h}$ of treatment.

\section{RNA isolation and semi-quantitative reverse transcription PCR}

Total RNA was isolated by Trizol reagent (Life Technologies, MD, USA). First-strand cDNA was synthesized according to the manufacturer's instructions (Invitrogen, CA, USA). The primer sets for DIRAS1 were designed to span intronic sequences between adjacent exons to control for genomic DNA contamination. Semi-quantitative reverse transcription PCR (RT-PCR) was amplified for 34 cycles. Glyceraldehyde-3-phosphatedehydrogenas (GAPDH) was used as an internal control. Primer sequences are shown in Additional file 1: Table S1.
Bisulfite modification, methylation-specific PCR, and bisulfite sequencing

DNA was prepared by the proteinase K method. In brief, cultured cells and fresh tissue samples were digested by DNA digestion buffer ( $\mathrm{pH}$ 8.0, $10 \mathrm{mM}$ Tris. $\mathrm{Cl}, 25 \mathrm{mM}$ EDTA, $1 \%$ SDS, $100 \mu \mathrm{g} / \mathrm{ml}$ proteinase $\mathrm{K}$ ) and extracted by phenol/chloroform. Bisulfite treatment was performed as previously described [14]. Methylation-specific PCR (MSP) primers were designed according to genomic sequences around transcriptional start sites (TSS) and synthesized to detect unmethylated (U) and methylated (M) alleles. Bisulfite sequencing (BSSQ) was performed as previously described [15]. BSSQ products were amplified by primers flanking the targeted regions including MSP products. Sequences of the MSP primers and bisulfite sequencing primers are shown in Additional file 1: Table S1. To obtain more evidence supporting our discovery, the expression and the methylation status of DIRAS1 in The Cancer Genome Atlas database were analyzed in primary colorectal cancer and adjacent tissue samples (Additional file 2: Figure S1).

\section{Immunohistochemistry}

Immunohistochemistry (IHC) was performed in primary colorectal cancer samples and paired adjacent tissue samples. The DIRAS1 antibody was diluted 1:100 (Bioworld Technology, Beijing, China). The staining intensity and extent of the staining area were scored using the German semi-quantitative scoring system as previously described [15].

\section{Plasmid construction and transfection}

Human full-length DIRAS1 coding sequences (CDS) was amplified and subcloned as described previously [4]. The primers used were 5'-CGCGGATCCATGCCGGAACA GAGTAACG-3' (F) and 5'-CCGCTCGAGTCACATGA GGGTGCATTTGC-3' (R). DIRAS1 expressing lentiviral or empty vectors were packaged using the ViraPower $^{\text {tw }}$ lentiviral expression system (Invitrogen, San Diego, CA, USA). Lentivirus was added to the growing medium of DLD1 and RKO cells, and DIRAS1 stably expressed cells (DLD1-DIRAS1 and RKO-DIRAS1 cells) and control cells (DLD1-Vector and RKO-Vector cells) were selected by blasticidin (Invitrogen, San Diego, CA, USA) at a concentration of $5 \mu \mathrm{g} / \mathrm{ml}$. The selected siRNAs targeting DIRAS1 sequences were as follows: sense: $5^{\prime}$-CCACCAGGCAA UAACCACATT-3'; antisense: 5'-UCGAUGUUGAGG CUCAUGUTT-3'; negative control sequences were as follows: sense: 5'-UUCUCCGAACGUGUCACGUTT-3'; antisense: 5' -ACGUGACACGUUCGGAGAATT-3'.

\section{Western blot}

Protein preparation and Western blot were performed as described previously [16]. The antibodies for Western 
blot analysis were as follows: rabbit anti-DIRAS1 (Bioworld Technology, Beijing, China), rabbit anti-MMP2 (Bioworld Technology, Beijing, China), rabbit antiMMP9 (Bioworld Technology, Beijing, China), and rabbit anti-cleaved caspase 3 (Bioworld Technology, Beijing, China). Rabbit anti-GAPDH (Bioworld Technology, Beijing, China) was used as a control.

\section{Cell viability detection}

Cells were plated into 96 -well plates at $1.5 \times 10^{3}$ cells/ well, and the cell viability was measured by the MTT assay (KeyGEN Biotech, Nanjing, China) at 0, 24, 48, and $72 \mathrm{~h}$. Absorbance was measured on a microplate reader (Thermo Multiskan MK3, MA, USA) at a wavelength of $490 \mathrm{~nm}$.

\section{Colony formation assay}

Cells were seeded at 500 cells per well in 6-well culture plates in triplicate. The complete growth medium conditioned with blasticidin at $2 \mu \mathrm{g} / \mathrm{ml}$ was exchanged every $48 \mathrm{~h}$. After 2 weeks, cells were fixed with $75 \%$ ethanol for $30 \mathrm{~min}$ and stained with $0.2 \%$ crystal violet (Beyotime, Nanjing, China) for visualization and counting.

\section{Flow cytometry}

DIRAS1 unexpressed and re-expressed DLD1 and RKO cells were treated with staurosporine (STS) at 100 and $120 \mathrm{ng} / \mathrm{ml}$, respectively, for $24 \mathrm{~h}$ [13]. The cells were prepared using the FITC Annexin V Apoptosis
Detection Kit I (BD Biosciences, Franklin Lakes, NJ, USA) following the manufacturer's instructions and then sorted by FACS Calibur (BD Biosciences, Franklin Lakes, NJ, USA).

\section{Transwell assay}

Cells were suspended in serum-free medium. Cells $(2 \times$ $10^{5}$ ) were seeded into the upper chamber of an $8-\mu \mathrm{m}$ pore size transwell apparatus (Corning, NY, USA) and incubated for $20 \mathrm{~h}$. Cells that migrated to the lower surface of the membrane were stained with crystal violet and counted in three independent fields. For invasion analysis, cells $\left(2 \times 10^{5}\right)$ were placed into the upper chamber of a transwell apparatus coated with extracellular matrix gel (ECM gel, BD Biosciences, San Jose, CA) and incubated for $48 \mathrm{~h}$. Cells that invaded into the lower membrane surface were stained with crystal violet and counted in three independent fields.

\section{Generation of colorectal cancer xenografts and assessment of tumor growth}

DLD1-DIRAS1 and control cells $\left(1 \times 10^{7}\right.$ cells in $200 \mu \mathrm{l}$ PBS) were injected subcutaneously into the dorsal right flank of male athymic nude mice. Each group includes eight mice. Subcutaneous tumor volumes $(V)$ were measured weekly with digital calipers and calculated using the formula $V=1 / 2 \times$ length $\times(\text { width })^{2}$. Mice were sacrificed on the 21st day; tumor weight was measured.

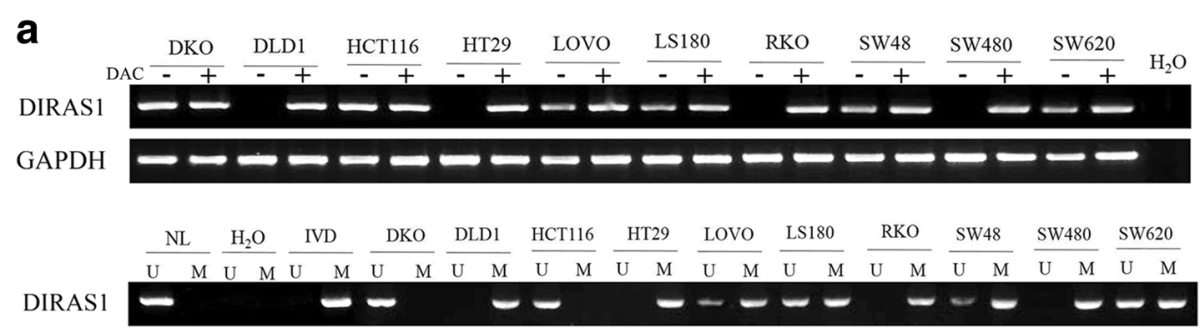

b

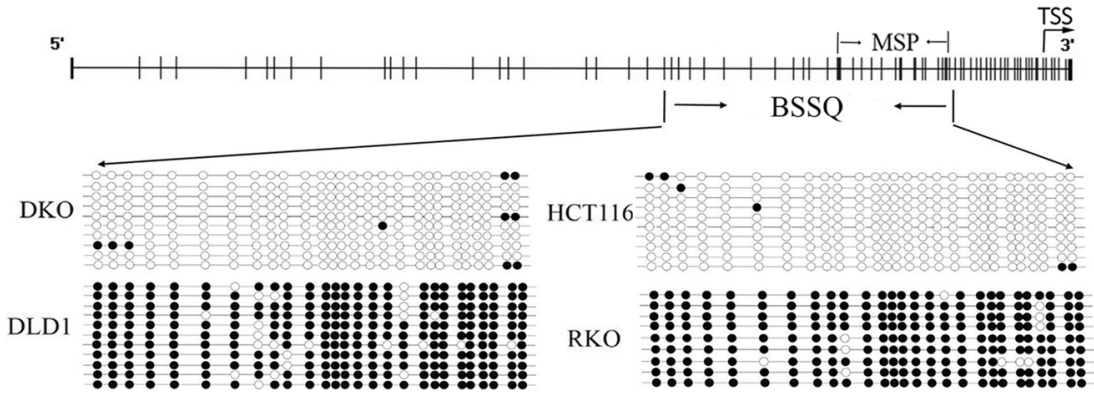

Fig. 1 The expression and methylation status of DIRAS1 in colorectal cancer cells. a Top panel: semi-quantitative RT-PCR shows DIRAS1 expression levels in colorectal cancer cell lines: DKO, DLD1, HCT116, HT29, LoVo, LS180, RKO, SW48, SW480, and SW620. DAC 5-aza-2'-deoxycytidine, GAPDH internal control of RT-PCR, $\mathrm{H}_{2} \mathrm{O}$ double distilled water. (-) absence of DAC. (+) presence of DAC. Bottom panel: MSP results of DIRAS1 in colorectal cancer cell lines. $U$ unmethylated alleles, $M$ methylated alleles, IVD in vitro methylated DNA, serves as methylation control, NL normal lymphocytes DNA, serves as unmethylation control. b BSSQ results of DIRAS1. MSP PCR product spanned 143 bp in DIRAS1 promoter region. Bisulfite sequencing region is located $284 \mathrm{bp}$ upstream of transcription start site in the genome. TSS transcription start site 


\section{Statistical analysis}

SPSS 18.0 software (IBM, NY, USA) was used for data analysis. All data were presented as means \pm standard deviation (SD) and analyzed using the Student's $t$ test. The Chi-squared test and the Fisher's exact test were used to analyze the association of DIRAS1 methylation and clinic-pathologic factors, as well as the association of DIRAS1 expression and the promoter region methylation. The association of DIRAS1 methylation and overall survival rate of patients were calculated by the KaplanMeier method, and differences in survival curve were evaluated using the log-rank test. The correlation between mRNA expression and methylation was performed with the Spearman's rank correlation coefficient (rho). The value of $P<0.05$ was considered to be a significant difference.

\section{Results}

DIRAS1 is silenced by promoter region hypermethylation in colorectal cancer cells

To explore the regulation of DIRAS1 in colorectal cancer, the expression of DIRAS1 was detected by semiquantitative RT-PCR in 10 colorectal cancer cell lines. DIRAS1 was expressed in DKO and HCT116 cells. Reduced expression was found in LoVo, SW48, LS180, and SW620 cells. No expression of DIRAS1 was detected in DLD1, HT29, RKO, and SW480 cells (Fig. 1a, top panel). Methylation was evaluated by methylation-specific PCR. Complete methylation was found in the promoter region of DLD1, HT29, RKO, and SW480 cells. Partial methylation was detected in LoVo, LS180, SW48, and SW620 cells, and unmethylation was found in DKO and HCT116 cells (Fig. 1a, bottom panel). Methylation results were

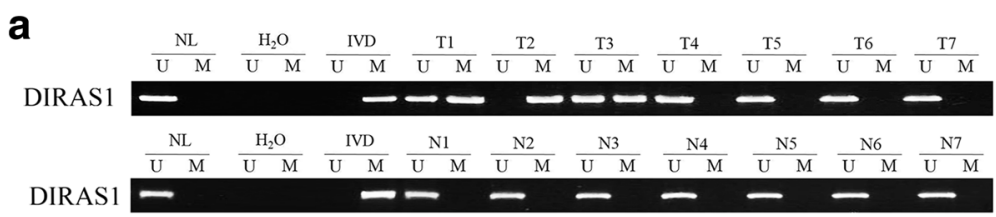

b

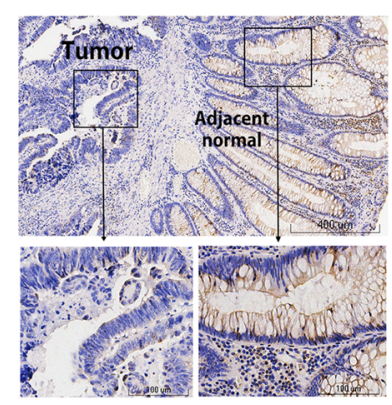

C

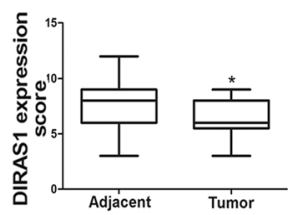

d

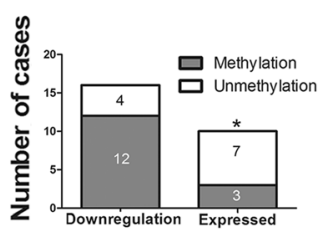

e

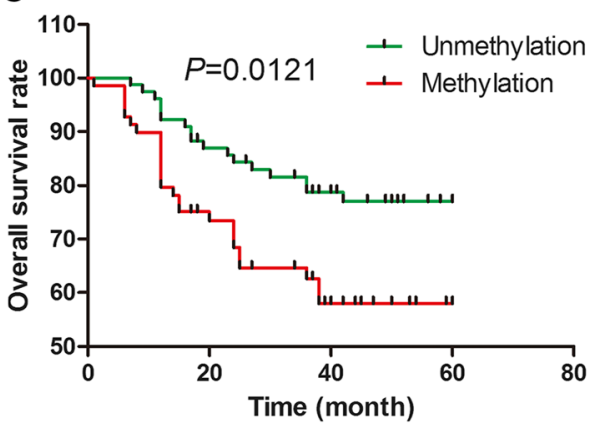

Fig. 2 Methylation status and expression of DIRAS1 in primary colorectal cancer samples. a Representative results of MSP for DIRAS1 in primary colorectal cancer samples and matched adjacent tissue samples. T primary colorectal cancer samples, $N$ normal colorectal mucosa. b Representative IHC results show the expression levels of DIRAS1 in colorectal cancer and matched adjacent tissue samples (upper: $\times 100 ;$ lower: $\times 400)$. c DIRAS1 expression scores are shown as box plots, horizontal lines represent the median score; the bottom and top of the boxes represent the 25th and 75th percentiles, respectively; vertical bars represent the range of data. The expression levels of DIRAS1 were significantly different between adjacent tissue and colorectal cancer samples. $\left.{ }^{*} P<0.05\right)$. $\mathbf{d}$ The bar diagram shows the expression and DNA methylation status of DIRAS1 in different cancer samples. Reduced expression of DIRAS1 was significantly associated with promoter region methylation. $\left({ }^{*} P<0.05\right)$. e Kaplan-Meier curves show the association of overall survival rate of colorectal cancer patients with the methylation status of DIRAS1. Green, DIRAS1 unmethylated colorectal cancer patients ( $n=77$, median survival, 25 months); red, DIRAS1 methylated colorectal cancer patients ( $n=69$, median survival, 51 months, $P=0.0121$, log-rank test) 
validated by bisulfite sequencing in DKO, HCT116, DLD1, and RKO cells (Fig. 1b). Promoter region methylation correlated with loss of/reduced expression of DIRAS1. Reexpression of DIRAS1 was induced by DAC, a demethylation agent, in DLD1, HT29, RKO, and SW480 cells (Fig. 1a, top panel). These results suggest that the expression of $D I R A S 1$ is regulated by promoter region methylation.

DIRAS1 is frequently methylated in human primary colorectal cancer, and methylation of DIRAS1 is associated with poor prognosis

Methylation of DIRAS1 was detected in $47.3 \%(69 / 146)$ of primary colorectal cancers, and no methylation was found in 50 cases of non-cancerous colonic tissue samples (Fig. 2a). The expression of DIRAS1 was evaluated by IHC in 26 matched cancer and adjacent tissue samples. Low-level expression of DIRAS1 was found in 16 of 26 (61.5\%) cancer samples compared to adjacent noncancerous tissue samples (Fig. 2b, c). In the 16 cancer samples with low level expression of DIRAS1, 12 cases were methylated. By contrast in the 10 cancer samples that had normal DIRAS1 expression levels, 3 cases were methylated. Reduced expression of DIRAS1 was significantly associated with promoter region methylation $(P<$ 0.05 , Fig. $2 \mathrm{~d}$ ). These results indicate that DIRAS1 expression is regulated by promoter methylation in human primary colorectal cancer. Methylation of DIRAS1 was significantly associated with TNM stage $(P<0.05$, Table 1$)$ and reduced 5-year overall survival $(P=0.0121$, Fig. 2e). These results demonstrate that methylation of DIRAS1 may serve as a marker of poor prognosis in human colorectal cancer.

By searching the Cancer Genome Atlas (TCGA) database (https://cancergenome.nih.gov/), 217 cases of primary colorectal cancer and 16 cases of adjacent tissue samples were found to have both DIRAS1 gene expression and methylation data, which were obtained from RNA sequencing and HMK450 methylation array, respectively. The promoter region methylation was inversely associated with DIRAS1 expression in colorectal cancer $(R=-0.278$, $P<0.0001$, Additional file 2: Figure S1B). The levels of DNA methylation were significantly higher in colorectal cancer samples compared to adjacent tissue samples $(P<$ 0.0001 , Additional file 2: Figure S1C). No association was found between 5-year overall survival and DIRAS1 expression $(P>0.05$, Additional file 2: Figure S1D or methylation $(P>0.05$, Additional file 2: Figure $\mathrm{S} 1 \mathrm{E})$. The reason is that TCGA data was collected from different institutes and their therapeutic regimen maybe different.

DIRAS1 inhibits cell proliferation, migration, and invasion in colorectal cancer

To evaluate the effects of DIRAS1 on cell proliferation, cell viability was detected by MTT and colony formation
Table 1 Association of DIRAS1 methylation with clinicopathologic features in colorectal tumor

\begin{tabular}{|c|c|c|c|}
\hline \multirow[t]{2}{*}{ Clinical factor } & \multicolumn{2}{|c|}{ DIRAS1 methylation status } & \multirow[t]{2}{*}{$P$ value } \\
\hline & Unmethylated & $\overline{\text { Methylated }}$ & \\
\hline Gender & & & 0.72 \\
\hline Male & 53 & 45 & \\
\hline Female & 24 & 24 & \\
\hline Age (year) & & & 0.51 \\
\hline$>60$ & 40 & 40 & \\
\hline$\leq 60$ & 37 & 29 & \\
\hline Tumor size $(\mathrm{cm})$ & & & 0.86 \\
\hline$>5$ & 51 & 44 & \\
\hline$\leq 5$ & 26 & 25 & \\
\hline \multicolumn{4}{|l|}{ Differentiation } \\
\hline Well & 1 & 1 & 0.88 \\
\hline Moderate & 60 & 44 & \\
\hline Poor & 16 & 24 & \\
\hline Tumor invasion & & & 0.12 \\
\hline $\mathrm{T}_{1}$ & 0 & 0 & \\
\hline $\mathrm{T}_{2}$ & 16 & 6 & \\
\hline$T_{3}$ & 58 & 59 & \\
\hline $\mathrm{T}_{4}$ & 3 & 4 & \\
\hline Lymph node metastasis & & & 0.07 \\
\hline $\mathrm{N}_{0}$ & 48 & 32 & \\
\hline $\mathrm{N}_{1}$ & 29 & 37 & \\
\hline TNM stage & & & $0.02^{*}$ \\
\hline$|-| \mid$ & 47 & 28 & \\
\hline III-IV & 30 & 41 & \\
\hline
\end{tabular}

Chi-square test; ${ }^{*} P<0.05$

assays. The OD values at $72 \mathrm{~h}$ were $0.806 \pm 0.011$ vs $0.6843 \pm 0.035$ in DLD1 cells $(P<0.01)$ and $0.768 \pm 0.004$ vs. $0.621 \pm 0.003(P<0.01)$ in RKO cells before and after restoration of DIRAS1 expression (Fig. 3a). The results demonstrated that DIRAS1 inhibits cell viability in colorectal cancer cells. The clone numbers were $110.0 \pm 5.8$ vs. $52.7 \pm 8.2$ in DLD1 cells $(P<0.01)$ and $80.0 \pm 1.7$ vs. $52.7 \pm 6.4$ in RKO cells $(P<0.05)$ before and after restoration of DIRAS1 expression (Fig. 3b). The results suggest that DIRAS1 suppresses colorectal cancer cell growth. To further validate above results, siRNA technique was employed in DIRAS1 highly expressed HCT116 cells. The OD values were $0.335 \pm 0.005$ vs. $0.4920 \pm 0.012$ in HCT116 cells before and after knockdown of DIRAS1 $(P<0.001)$ (Fig. 4a). The results suggest that DIRAS1 inhibits cell viability in CRC cells. The clone numbers were $25.7 \pm 3.0$ vs. $48.0 \pm 3.6$ in HCT116 cells before and after knockdown of DIRAS1 in HCT116 cells $(P<0.01$, Fig. $4 \mathrm{~b}, \mathrm{c})$. It suggests that DIRAS1 suppresses colorectal cancer cell growth. 


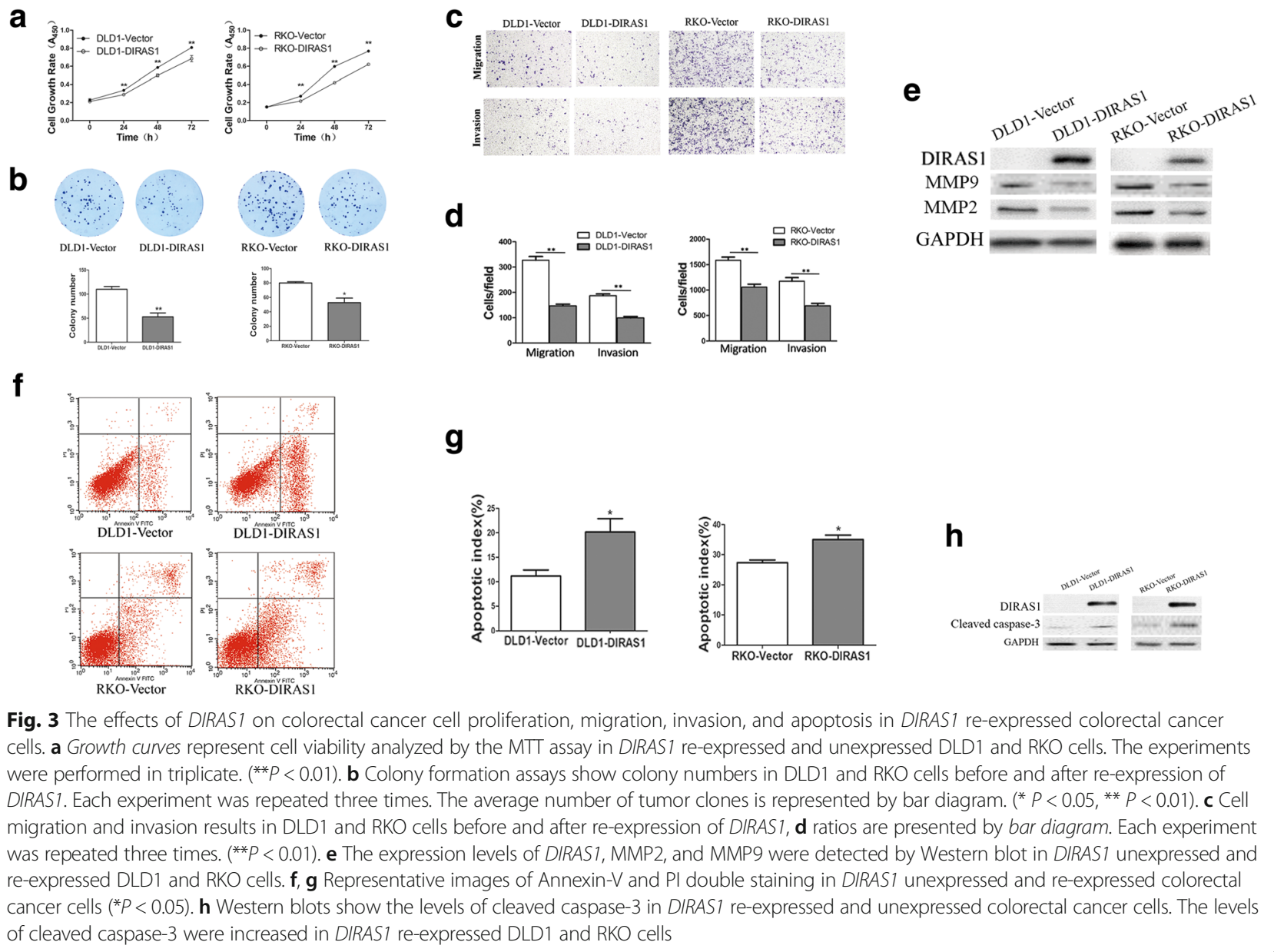

To explore the effect of DIRAS1 on cell migration, the transwell assay in the absence of ECM gel coating was employed. The numbers of migrated cells for each highpower field under the microscope were $327.0 \pm 14.8$ vs. $147.0 \pm 6.4$ in DLD1 cells $(P<0.01)$ and $1586.0 \pm 62.5$ vs. $1058.0 \pm 58.61$ in $\mathrm{RKO}$ cells $(P<0.01)$ before and after restoration of DIRAS1 expression (Fig. 3c top panel, Fig. 3d). The results demonstrate that DIRAS1 inhibits colorectal cancer cell migration. Next, the transwell assay with ECM coating was employed to evaluate the effect of DIRAS1 on cell invasion. The numbers of invasive cells for each high-power field under the microscope were $187.3 \pm 6.5$ vs. $99.7 \pm 5.2$ in DLD1 cells $(P<0.01)$ and $1173.0 \pm 71.2$ vs. $691.7 \pm 45.3$ in RKO cells $(P<0.01)$ before and after restoration of DIRAS1 expression (Fig. 3c bottom panel, Fig. 3d).

In DIRAS1 highly expressed HCT116 cells, siRNA technique was employed for transwell study. The numbers of migrated cells for each high-power field under the microscope were $1134.0 \pm 75.16$ vs. $1605.0 \pm 40.37$ $(P<0.01)$ cells before and after knockdown of DIRAS1 in HCT116 cells (Fig. 4d top panel, Fig. 4e). It further suggests that DIRAS1 inhibits cell migration in CRC. The numbers of invasive cells for each high-power field under the microscope were $428.7 \pm 19.6$ vs. $1329 \pm 54.0$ before and after knockdown of DIRAS1 in HCT116 cells $(P<0.001)$ (Fig. $4 \mathrm{~d}$ bottom panel, Fig. 4e). The result suggests that DIRAS1 inhibits cell invasion in colorectal cancer.

The expression levels of MMP2 and MMP9 were inhibited by re-expression of DIRAS1 in DLD1 and RKO cells (Fig. 3e), while they were increased after knockdown of DIRAS1 in DIRAS1 expressing HCT116 cells (Fig. 4f). These results suggest that DIRAS1 impedes colorectal cancer cell invasion.

DIRAS1-induced apoptosis in human colorectal cancer cells The effects of DIRAS1 on cell cycle and apoptosis were analyzed by flow cytometry in human colorectal cancer cells. No significant differences in cell phase distribution were found in DLD1 and RKO cells before and after reexpression of DIRAS1 (all $P>0.05$, data not shown). The ratios of apoptosis were $11.16 \pm 1.25 \%$ vs. $20.15 \pm 2.75 \%$ in DLD1 cells $(P<0.05)$ and $28.92 \pm 2.78 \%$ vs. $38.12 \pm 0.87 \%$ 
a

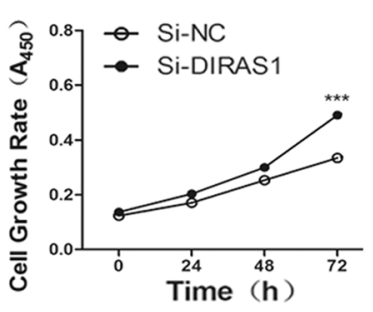

d

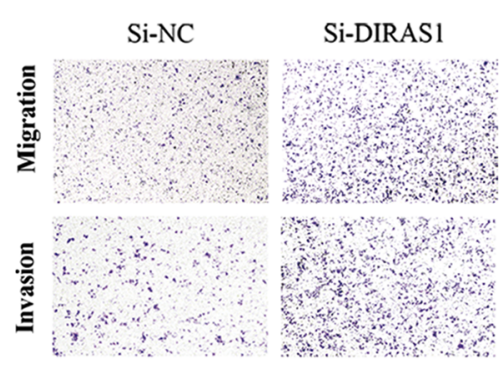

g

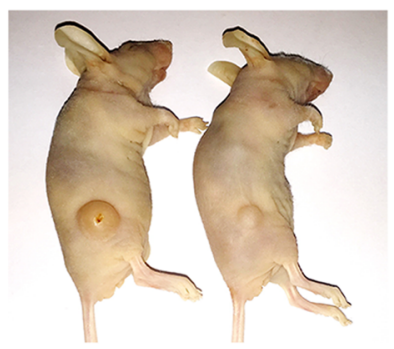

DLD1-Vector DLD1-DIRAS1

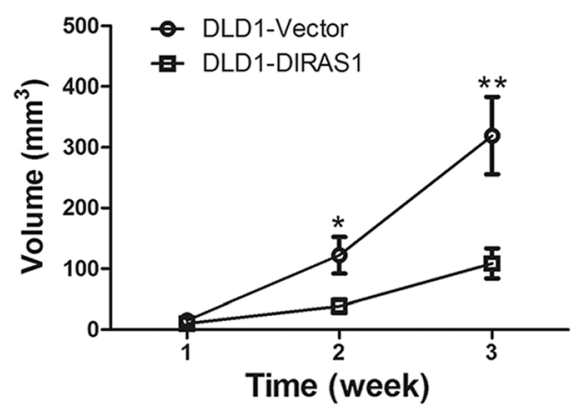

b
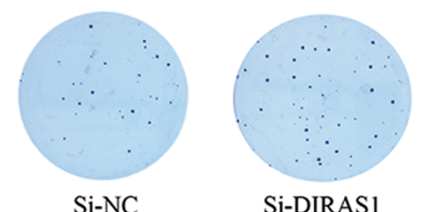

Si-DIRAS1
C

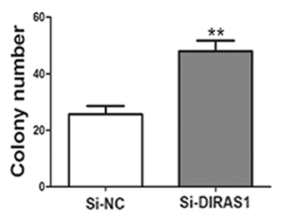

e

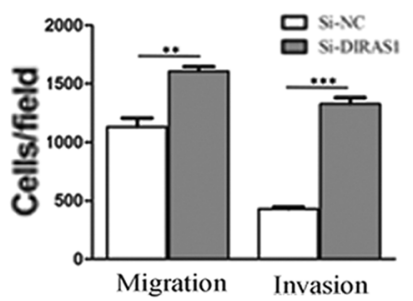

f

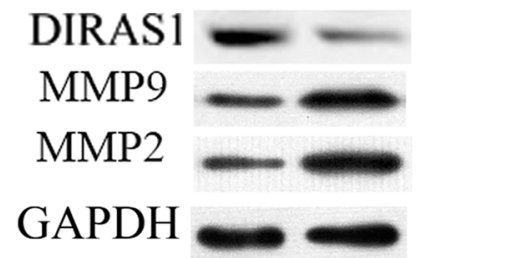

Fig. 4 The function of DIRAS1 in DIRAS1 highly expressed HCT116 cells and in vivo study of DIRAS1 in colorectal cancer cell xenograft mice. a Growth curves represent cell viability analyzed and by the MTT assay in DIRAS1 highly expressed HCT116 cells before and after knockdown of DIRAS1. The experiments were performed in triplicate. $\left.{ }^{* *} P<0.001\right)$. b Colony formation assays show colony numbers in DIRAS1 highly expressed HCT116 cells before and after knockdown of DIRAS1. Each experiment was repeated three times, $\mathbf{c}$ the average number of tumor clones is represented by bar diagram. (** $P<0.01)$. d Cell migration and invasion results in DIRAS1 highly expressed HCT116 cells before and after knockdown of DIRAS1, e ratios are presented by bar diagram. Each experiment was repeated three times. $\left(* * P<0.01,{ }^{* * *} P<0.001\right)$. f The expression levels of DIRAS1, MMP2, and MMP9 were detected by Western blot in DIRAS1 highly expressed HCT116 cells before and after knockdown of DIRAS1. Knockdown of DIRAS1 by siRNA was performed to validate the results in DIRAS1 highly expressing HCT116 cells. siNC DIRAS1 highly expressed HCT116 cells, siDIRAS1 knockdown of DIRAS1 in HCT116 cells. $\mathbf{g}$ Volumes of xenograft tumors in DIRAS1 re-expressed and unexpressed in DLD1 cells after 2 and 3 weeks, respectively $\left({ }^{*} P<0.05,{ }^{* *} P<0.01\right)$. $\mathbf{h}$ Weights of xenograft tumors in DIRAS1 re-expressed and unexpressed DLD1 and RKO cells after incubation for 3 weeks (**P<0.01). i IHC shows the expression of DIRAS1 in DIRAS1 re-expressed and unexpressed DLD1 cell xenografts $(\times 400)$ 
in RKO cells $(P<0.05)$ before and after re-expression of DIRAS1 under the treatment of STS (Fig. $3 \mathrm{f}, \mathrm{g}$ ). As shown in Fig. $3 \mathrm{~h}$, the levels of cleaved caspase- 3 were increased after re-expression of DIRAS1 in DLD1 and RKO cells.

\section{DIRAS1 suppresses tumor growth in xenograft mice}

To further validate the role of DIRAS1 in colorectal cancer, DIRAS1 unexpressed and re-expressed DLD1 cells were used to establish xenograft tumors in mice. The tumor volumes were $319.5 \pm 64.0 \mathrm{~mm}^{3}$ in DIRAS1 unexpressed DLD1 cell xenograft mice and 108.6 \pm $24.78 \mathrm{~mm}^{3}$ in DIRAS1 re-expressed DLD1 cell xenograft mice. The tumor volumes were smaller in DIRAS1 re-expressed DLD1 cell xenograft mice compared to DIRAS1 unexpressed DLD1 cell xenograft mice $(P<$ 0.01 , Fig. $4 \mathrm{~g})$. The tumor weights were $241.0 \pm 48.9 \mathrm{mg}$ in DIRAS1 unexpressed DLD1 cell xenograft mice and $72.7 \pm 21.6 \mathrm{mg}$ in DIRAS1 re-expressed DLD1 cell xenograft mice $(P<0.01$, Fig. 4 h). The tumor weights were lower in DIRAS1 expressed DLD1 cell xenograft mice compared to DIRAS1 unexpressed DLD1 cell xenograft mice. These results suggest that DIRAS1 suppressed colorectal cancer cell growth in vivo.

\section{Discussion}

The Ras superfamily is divided into five subfamilies according to their sequence homology and biochemical properties. The five families are Ras, Rab, Rho, Ran, and Arf. Ras members may activate downstream signaling by binding to GTP or serve as an inactive form by binding to GDP. When bound to GTP, Ras proteins associate with effectors, resulting in the propagation of downstream signaling. Most members of this superfamily have been widely studied and identified as oncoproteins [17, 18]. DIRAS1 may serve as a competitive inhibitor of Ras and antagonize Ras-mediated ERK1/2 signaling to promote cell apoptosis and suppress cell invasion. The expression levels of DIRAS1 were found to be reduced in human breast cancer and esophageal cancer $[13,19]$. In this study, we found that DIRAS1 is frequently methylated in human $\mathrm{CRC}$, and the expression of DIRAS1 is regulated by promoter region methylation. In TCGA database, the promoter region methylation was inversely associated with DIRAS1 expression in colorectal cancer. The levels of DNA methylation were significantly higher in colorectal cancer samples compared to adjacent tissue samples according to HMK450 methylation array data in human colorectal cancer. These data further supported our results. Methylation of DIRAS1 was associated with TNM stage and short survival. These results suggest that methylation of DIRAS1 may serve as a marker of poor prognosis in human colorectal cancer. In our studied cohort, patients were received different chemotherapeutic regimens. It limits to further analyze the association of DIRAS1 methylation and each chemical agent or therapy. The consequence of each regimen may be different in CRC treatment, and TCGA data was collected from different institutes by using different regimens. It may affect to evaluating the association between the 5-year overall survival and DIRAS1 expression or methylation. A welldesigned large cohort study is necessary to further validate that DIRAS1 methylation is a marker of poor prognosis in $\mathrm{CRC}$ by using a unique detection method.

DIRAS1 inhibits cell proliferation, migration, and invasion and induces apoptosis in colorectal cancer cells. DIRAS1 suppresses colorectal cancer cell xenograft growth in vivo. Collectively, our results suggest that DIRAS1 is a tumor suppressor in human CRC.

\section{Conclusions}

DIRAS1 is frequently methylated in human colorectal cancer, and the expression of DIRAS1 is regulated by promoter region methylation. Methylation of DIRAS1 is a marker of poor prognosis in human colorectal cancer. Methylation of DIRAS1 may promote colorectal carcinogenesis and progression.

\section{Additional files}

Additional file 1: Table S1. Nucleotide sequences of the primers used
in this study. (XLS $25 \mathrm{~kb})$
Additional file 2: Figure S1. Methylation status and expression of
DIRAS1 in primary colorectal cancer and adjacent samples from TCGA. (A)
The correlation of methylation of each CpG site (HM450) and expression
of DIRAS1. (B) The methylation status of the CpG site (cg05228284, HM450)
is correlated to loss of/reduced DIRAS1 expression in 217 cases of colorectal
cancer samples and 16 cases of adjacent samples. ( $R=-0.278, P<0.0001$ ).
(C) The methylation status of the CpG site (cg05228284, HM450) is
correlated to loss of/reduced DIRAS1 expression in 234 cases of colorectal
cancer. ( $P<0.0001)$. (D) Kaplan-Meier curves show the association of overall
survival rate of colorectal cancer patients with the methylation status of
CpG site (cg05228284, HM450). ( $P>0.05)$. (E) Kaplan-Meier curves show the
association of overall survival rate of colorectal cancer patients with the
expression levels of DIRAS1. ( $P>0.05)$. (TIF 572 kb)

\section{Abbreviations}

BSSQ: Bisulfite sequencing; CRC: Colorectal cancer; DAC: 5-Aza-2'-deoxycytidine; DKO: DNMT1 and DNMT3b double knockout; ECM: Extracellular matrix; GAPDH: Glyceraldehyde-3-phosphatedehydrogenas; IHC: Immunohistochemistry; M: Methylated; MSP: Methylation-specific PCR; Rig: Ras-related inhibitor of cell growth; SD: Standard deviation; STS: Staurosporine; TSS: Transcriptional start sites; U: Unmethylated

\section{Acknowledgements}

We sincerely thank Xiaomo Su for preparing experiments.

\section{Funding}

This work was supported by grants from the National Basic Research Program of China (973 Program No. 2012CB934002), National Key Research and Development Programme of China (2016YFC1303600); National Key Scientific Instrument Special Programme of China (Grant No. 2011YQ03013405); National Science Foundation of China (NSFC Nos. 8167100001, 81402345, U1604281, 81672318); Beijing Science Foundation of China (BJSFC No. 7171008). 


\section{Availability of data and materials}

The datasets analyzed during the current study available from the corresponding author on reasonable request.

\section{Authors' contributions}

RZ performed the research and analyzed the data. RZ and MG wrote the manuscript. MG made substantial contributions to conception and coordination of the study. DG, TH and MZ help collecting samples. XZ and EL provided manuscript and experimental advices. LW supervised the study. All authors read and approved the final manuscript.

\section{Competing interests}

The authors declare that they have no competing interests.

\section{Consent for publication}

Not applicable

\section{Ethics approval and consent to participate}

This study was approved by the institutional review board of the Chinese PLA General Hospital.

\section{Publisher's Note}

Springer Nature remains neutral with regard to jurisdictional claims in published maps and institutional affiliations.

\section{Author details}

'Department of Gastroenterology and Hepatology, Chinese People's Liberation Army General Hospital, \#28 Fuxing Road, Beijing 100853, China. 2Department of Pathology, Chinese People's Liberation Army General Hospital, 28 Fu-Xing Road, Beijing 100853, China. ${ }^{3}$ School of Medicine, Nankai University, \#94 Weijin Road, Tianjin 300071, China.

Received: 18 January 2017 Accepted: 26 April 2017

Published online: 10 May 2017

\section{References}

1. Fitzmaurice C, Allen C, Barber RM, Barregard L, Bhutta ZA, et al. Global, regional, and national cancer incidence, mortality, years of life lost, years lived with disability, and disability-adjusted life-years for 32 cancer groups, 1990 to 2015: A Systematic Analysis for the Global Burden of Disease Study. JAMA Oncol. 2017;3(4):524-48.

2. Fitzmaurice $C$, Dicker D, Pain A, Hamavid H, Moradi-Lakeh $M$, et al. The global burden of cancer 2013. JAMA Oncol. 2015;1:505-27.

3. Jemal A. Global burden of cancer: opportunities for prevention. Lancet. 2012;380(9856):1797-9.

4. Okugawa Y, Grady WM, Goel A. Epigenetic alterations in colorectal cancer: emerging biomarkers. Gastroenterology. 2015;149(5):1204-25. e1212.

5. Burn J, Mathers J, Bishop DT. Genetics, inheritance and strategies for prevention in populations at high risk of colorectal cancer (CRC). Recent Results Cancer Res. 2013;191:157-83.

6. Wheeler JM, Loukola A, Aaltonen LA, Mortensen NJ, Bodmer WF. The role of hypermethylation of the hMLH1 promoter region in HNPCC versus MSI+ sporadic colorectal cancers. J Med Genet. 2000;37(8):588-92.

7. Haydon AM, Jass JR. Emerging pathways in colorectal-cancer development. Lancet Oncol. 2002;3(2):83-8.

8. Gao D, Herman JG, Guo M. The clinical value of aberrant epigenetic changes of DNA damage repair genes in human cancer. Oncotarget. 2016; 7(24):37331-46.

9. Hu S, Cao B, Zhang M, Linghu E, Zhan Q, Brock MV, Herman JG, Mao G, Guo M. Epigenetic silencing BCL6B induced colorectal cancer proliferation and metastasis by inhibiting P53 signaling. Am J Cancer Res. 2015;5(2):651-62.

10. Guo J, Yang Y, Yang Y, Linghu E, Zhan Q, Brock MV, Herman JG, Zhang B, Guo M. RASSF10 suppresses colorectal cancer growth by activating P53 signaling and sensitizes colorectal cancer cell to docetaxel. Oncotarget. 2015;6(6):4202-13.

11. Cao B, Yang Y, Pan Y, Jia Y, Brock MV, Herman JG and Guo M. Epigenetic silencing of CXCL14 induced colorectal cancer migration and invasion. Discovery medicine. 2013;16(88):137-47.

12. Ellis CA, Vos MD, Howell H, Vallecorsa T, Fults DW, Clark GJ. Rig is a novel Ras-related protein and potential neural tumor suppressor. Proc Natl Acad Sci U S A. 2002;99(15):9876-81.
13. Zhu YH, Fu L, Chen L, Qin YR, Liu H, Xie F, Zeng T, Dong SS, Li J, Li Y, Dai Y, Xie D, Guan XY. Downregulation of the novel tumor suppressor DIRAS1 predicts poor prognosis in colorectal squamous cell carcinoma. Cancer Res. 2013;73(7):2298-309.

14. Herman JG, Graff JR, Myohanen S, Nelkin BD, Baylin SB. Methylation-specific PCR: a novel PCR assay for methylation status of CpG islands. Proc Natl Acad Sci U S A. 1996;93(18):9821-6.

15. Jia Y, Yang Y, Liu S, Herman JG, Lu F, Guo M. SOX17 antagonizes WNT/ beta-catenin signaling pathway in hepatocellular carcinoma. Epigenetics. 2010;5(8):743-9.

16. Yan W, Wu K, Herman JG, Brock MV, Fuks F, Yang L, Zhu H, Li Y, Yang Y, Guo M. Epigenetic regulation of DACH1, a novel Wnt signaling component in colorectal cancer. Epigenetics. 2013;8(12):1373-83.

17. Reuther GW, Der CJ. The Ras branch of small GTPases: Ras family members don't fall far from the tree. Curr Opin Cell Biol. 2000;12(2):157-65.

18. Zohn IM, Campbell SL, Khosravi-Far R, Rossman KL, Der CJ. Rho family proteins and Ras transformation: the RHOad less traveled gets congested. Oncogene. 1998;17(11 Reviews):1415-38.

19. Bergom C, Hauser AD, Rymaszewski A, Gonyo P, Prokop JW, Jennings BC, Lawton AJ, Frei A, Lorimer EL, Aguilera-Barrantes I, Mackinnon AC, Noon K, Fierke CA, Williams CL. The tumor-suppressive small GTPase DIRAS1 binds the noncanonical guanine nucleotide exchange factor SmgGDS and antagonizes SmgGDS interactions with oncogenic small GTPases. J Biol Chem. 2016:291(12):6534-45.

\section{Submit your next manuscript to BioMed Central and we will help you at every step:}

- We accept pre-submission inquiries

- Our selector tool helps you to find the most relevant journal

- We provide round the clock customer support

- Convenient online submission

- Thorough peer review

- Inclusion in PubMed and all major indexing services

- Maximum visibility for your research

Submit your manuscript at www.biomedcentral.com/submit
Biomed Central 